Louisiana State University

LSU Digital Commons

Faculty Publications

Department of Physics \& Astronomy

2-1-2011

\title{
When a standard candle flickers
}

\author{
Colleen A. Wilson-Hodge \\ NASA Marshall Space Flight Center \\ Michael L. Cherry \\ Louisiana State University \\ Gary L. Case \\ Louisiana State University \\ Wayne H. Baumgartner \\ National Aeronautics and Space Administration \\ Elif Beklen \\ Middle East Technical University (METU)
}

See next page for additional authors

Follow this and additional works at: https://digitalcommons.Isu.edu/physics_astronomy_pubs

\section{Recommended Citation}

Wilson-Hodge, C., Cherry, M., Case, G., Baumgartner, W., Beklen, E., Bhat, P., Briggs, M., Camero-Arranz, A., Chaplin, V., Connaughton, V., Finger, M., Gehrels, N., Greiner, J., Jahoda, K., Jenke, P., Kippen, R., Kouveliotou, C., Krimm, H., Kuulkers, E., Lund, N., Meegan, C., Natalucci, L., Paciesas, W., Preece, R., Rodi, J., Shaposhnikov, N., Skinner, G., Swartz, D., Von Kienlin, A., Diehl, R., \& Zhang, X. (2011). When a standard candle flickers. Astrophysical Journal Letters, 727 (2 PART II) https://doi.org/10.1088/2041-8205/727/2/ L40

This Article is brought to you for free and open access by the Department of Physics \& Astronomy at LSU Digital Commons. It has been accepted for inclusion in Faculty Publications by an authorized administrator of LSU Digital Commons. For more information, please contact ir@lsu.edu. 


\section{Authors}

Colleen A. Wilson-Hodge, Michael L. Cherry, Gary L. Case, Wayne H. Baumgartner, Elif Beklen, P. Narayana Bhat, Michael S. Briggs, Ascension Camero-Arranz, Vandiver Chaplin, Valerie Connaughton, Mark H.

Finger, Neil Gehrels, Jochen Greiner, Keith Jahoda, Peter Jenke, R. Marc Kippen, Chryssa Kouveliotou, Hans A. Krimm, Erik Kuulkers, Niels Lund, Charles A. Meegan, Lorenzo Natalucci, William S. Paciesas,

Robert Preece, James C. Rodi, Nikolai Shaposhnikov, Gerald K. Skinner, Doug Swartz, Andreas Von Kienlin, Roland Diehl, and Xiao Ling Zhang 


\section{When a Standard Candle Flickers}

Wilson-Hodge, Colleen A; Cherry, Michael L; Case, Gary L; Baumgartner, Wayne H; Beklen, Elif; Bhat, P Narayana; Briggs, Michael S; Camero-Arranz, Ascension; Chaplin, Vandiver; Connaughton, Valerie Total number of authors:

31

Published in:

Astrophysical Journal Letters

Link to article, DOI:

10.1088/2041-8205/727/2/L40

Publication date:

2011

Document Version

Publisher's PDF, also known as Version of record

Link back to DTU Orbit

Citation (APA):

Wilson-Hodge, C. A., Cherry, M. L., Case, G. L., Baumgartner, W. H., Beklen, E., Bhat, P. N., Briggs, M. S., Camero-Arranz, A., Chaplin, V., Connaughton, V., Finger, M. H., Gehrels, N., Greiner, J., Jahoda, K., Jenke, P., Kippen, R. M., Kouveliotou, C., Krimm, H. A., Kuulkers, E., ... Zhang, X-L. (2011). When a Standard Candle Flickers. Astrophysical Journal Letters, 727(2), L40. https://doi.org/10.1088/2041-8205/727/2/L40

\section{General rights}

Copyright and moral rights for the publications made accessible in the public portal are retained by the authors and/or other copyright owners and it is a condition of accessing publications that users recognise and abide by the legal requirements associated with these rights.

- Users may download and print one copy of any publication from the public portal for the purpose of private study or research.

- You may not further distribute the material or use it for any profit-making activity or commercial gain

- You may freely distribute the URL identifying the publication in the public portal 


\title{
WHEN A STANDARD CANDLE FLICKERS
}

\author{
Colleen A. Wilson-Hodge ${ }^{1}$, Michael L. Cherry ${ }^{2}$, Gary L. Case ${ }^{2}$, Wayne H. Baumgartner ${ }^{3}$, Elif Beklen ${ }^{4,5}$, \\ P. Narayana Bhat ${ }^{6}$, Michael S. Briggs ${ }^{6}$, Ascension Camero-Arranz ${ }^{7}$, Vandiver Chaplin $^{6}$, Valerie Connaughton $^{6}$, \\ Mark H. Finger ${ }^{8}$, Neil Gehrels ${ }^{9}$, Jochen Greiner ${ }^{10}$, Keith Jahoda ${ }^{9}$, Peter Jenke ${ }^{1,17}$, R. Marc Kippen ${ }^{11}$, \\ Chryssa Kouveliotou ${ }^{1}$, Hans A. Krimm ${ }^{3,12}$, Erik Kuulkers ${ }^{13}$, Niels Lund ${ }^{14}$, Charles A. Meegan ${ }^{8}$, Lorenzo Natalucci ${ }^{15}$, \\ William S. Paciesas ${ }^{6}$, Robert Preece ${ }^{6}$, James C. Rodi ${ }^{2}$, Nikolai Shaposhnikov ${ }^{3,16}$, Gerald K. Skinner ${ }^{3,16}$, Doug Swartz ${ }^{8}$, \\ AndReas von Kienlin ${ }^{10}$, Roland Diehl ${ }^{10}$, and XiaO-Ling Zhang ${ }^{10}$ \\ ${ }^{1}$ VP 62 Space Science Office, NASA Marshall Space Flight Center, Huntsville, AL 35812, USA; colleen.wilson@ nasa.gov \\ ${ }^{2}$ Department of Physics and Astronomy, Louisiana State University, Baton Rouge, LA 70803, USA \\ ${ }^{3}$ CRESST/NASA GSFC, Astrophysics Science Division, Greenbelt, MD 20771, USA \\ ${ }^{4}$ Physics Department, Middle East Technical University, 06531 Ankara, Turkey \\ ${ }^{5}$ Physics Department, Suleyman Demirel University, 32260 Isparta, Turkey \\ ${ }^{6}$ University of Alabama in Huntsville, Huntsville, AL 35899, USA \\ ${ }^{7}$ National Space Science and Technology Center, Huntsville, AL 35805, USA \\ ${ }^{8}$ Universities Space Research Association, Huntsville, AL 35805, USA \\ ${ }^{9}$ NASA Goddard Space Flight Center (GSFC), Greenbelt, MD 20771, USA \\ ${ }^{10}$ Max-Planck Institut für Extraterrestische Physik, 85748 Garching, Germany \\ ${ }^{11}$ Los Alamos National Laboratory, Los Alamos, NM 87545, USA \\ 12 Universities Space Research Association, Columbia, MD 21044, USA \\ ${ }^{13}$ ISOC, ESA, European Space Astronomy Centre (ESAC), P.O. Box 78, 28691 Villanueva de la Cañada (Madrid), Spain \\ ${ }^{14}$ Danish National Space Center, Technical University of Denmark, Juliane Maries Vej 30, 2100 Copenhagen, Denmark \\ ${ }^{15}$ INAF-IASF Roma, via Fosso del Cavaliere 100, 00133 Roma, Italy \\ ${ }^{16}$ Astronomy Department, University of Maryland, College Park, MD 20742, USA \\ Received 2010 September 24; accepted 2010 November 17; published 2011 January 12
}

\begin{abstract}
The Crab Nebula is the only hard X-ray source in the sky that is both bright enough and steady enough to be easily used as a standard candle. As a result, it has been used as a normalization standard by most X-ray/gamma-ray telescopes. Although small-scale variations in the nebula are well known, since the start of science operations of the Fermi Gamma-ray Burst Monitor (GBM) in 2008 August, a 7\% (70 mCrab) decline has been observed in the overall Crab Nebula flux in the $15-50 \mathrm{keV}$ band, measured with the Earth occultation technique. This decline is independently confirmed in the $\sim 15-50 \mathrm{keV}$ band with three other instruments: the Swift Burst Alert Telescope (Swift/BAT), the Rossi X-ray Timing Explorer Proportional Counter Array (RXTE/PCA), and the Imager on-Board the INTEGRAL Satellite (IBIS). A similar decline is also observed in the $\sim 3-15 \mathrm{keV}$ data from the RXTE/PCA and in the 50-100 keV band with GBM, Swift/BAT, and INTEGRAL/IBIS. The pulsed flux measured with RXTE/PCA since 1999 is consistent with the pulsar spin-down, indicating that the observed changes are nebular. Correlated variations in the Crab Nebula flux on a $\sim 3$ year timescale are also seen independently with the PCA, BAT, and IBIS from 2005 to 2008, with a flux minimum in 2007 April. As of 2010 August, the current flux has declined below the 2007 minimum.
\end{abstract}

Key words: pulsars: individual (Crab Pulsar) - X-rays: individual (Crab Nebula)

\section{INTRODUCTION}

$\mathrm{X}$-ray and gamma-ray astronomers frequently consider the Crab supernova remnant to be a steady standard candle suitable as a calibration source (e.g., Kirsch et al. 2005; Jourdain \& Roques 2009; Weisskopf et al. 2010; Meyer et al. 2010). Jourdain \& Roques (2009) presented over five years of the Spectrometer on INTEGRAL (SPI, $20 \mathrm{keV}-8 \mathrm{MeV}$ ) observations, with fitted flux normalizations at $100 \mathrm{keV}$ consistent with being constant to within the $\sim 3 \%$ quoted errors. On the basis of data from XMM-Newton, INTEGRAL, Swift, Chandra, RXTE, and several earlier missions, Kirsch et al. (2005) have concluded that the Crab flux can be described at least up to $30 \mathrm{keV}$ by the same spectrum proposed by Toor \& Seward (1974) three decades earlier: $d N / d E=(9.7 \pm 1.0) E^{-(2.1 \pm 0.03)}$ photons $\mathrm{cm}^{-2} \mathrm{~s}^{-1} \mathrm{keV}^{-1}$, i.e., they describe the $\mathrm{Crab}$ as a standard candle.

Driven by the central pulsar's spin-down luminosity, the surrounding remnant consists of a cloud of expanding thermal

\footnotetext{
${ }^{17}$ NASA Postdoctoral Program Fellow.
}

ejecta and a synchrotron nebula (Hester 2008) with an integrated luminosity $\sim 10^{38} \mathrm{erg} \mathrm{s}^{-1}$. The pulsar provides a shocked wind that accelerates electrons and positrons to energies $\sim 10^{7} \mathrm{GeV}$ and a source of kinetic energy driving turbulent motion of a ring of wisps nearly surrounding the synchrotron nebula. High-resolution observations reveal wisps and knots moving at velocities up to $0.7 c$ from radio to $\mathrm{X}$-ray energies (Hester et al. 1995, 2002; Greiveldinger \& Aschenbach 1999; Bietenholz et al. 2001; Mori et al. 2004). A central torus and jet structure extending out from the pulsar were observed in X-rays by Chandra (Weisskopf et al. 2000), aligned closely with the pulsar's proper motion (Ng \& Romani 2006). The nebular emission is considered to be a combination of synchrotron radiation up to $\sim 100 \mathrm{MeV}$ and a harder inverse Compton spectrum extending up to $\mathrm{TeV}$ energies (De Jager et al. 1996).

Observations of the $8 \mathrm{GHz}$ nebular flux in 1985 (Aller \& Reynolds 1985) showed a decrease of $0.167 \% \pm 0.015 \% \mathrm{yr}^{-1}$, consistent with predictions by Reynolds \& Chevalier (1984) for an expanding synchrotron-emitting cloud. At optical wavelengths, Smith (2003) reported a decrease in the 
nebula-integrated flux of $0.5 \% \pm 0.2 \% \mathrm{yr}^{-1}$ from 1987 to 2002 . At X-ray energies (2-28 keV), 1996-2002 BeppoSAX observations described by Verrecchia et al. (2007) included a $2 \%$ systematic error to account for the observed fluctuations in time. In the 35-300 keV energy region, Ling \& Wheaton (2003) reported $\sim 10 \%$ variations in the flux observed with the Burst and Transient Source Experiment (BATSE) on the Compton Gamma-Ray Observatory (CGRO) over periods of days to weeks.

Much et al. (1995) reported a $\sim 40 \%$ increase in the unpulsed flux (0.75-30 MeV) measured with the CGRO Compton Telescope (COMPTEL) between 1991 April/May and 1992 August/September. At the same time, De Jager et al. (1996) reported a $\sim 50 \%$ decrease in the $75-150 \mathrm{MeV}$ flux and steady emission from $150 \mathrm{MeV}$ to $30 \mathrm{GeV}$ measured with the CGRO Energetic Gamma-Ray Experiment Telescope (EGRET) between 1991 and 1993. They interpret this as steady Compton emission $>150 \mathrm{MeV}$ from long-lived $\sim 5-100 \mathrm{GeV}$ electrons and $<150 \mathrm{MeV}$ synchrotron emission from shorter-lived $100 \mathrm{TeV}$ to $1 \mathrm{PeV}$ electrons accelerated by time-variable small-scale shock structures. A change in this electron acceleration mechanism would drop a portion of the electrons from the range responsible for the EGRET emission to the COMPTEL range, resulting in the observed fluxes. The Fermi Large Area Telescope (LAT) found no variations with time in the $100 \mathrm{MeV}-30 \mathrm{GeV}$ band from 2008 August to 2009 April (Abdo et al. 2010a). Recently, Fermi LAT (Abdo et al. 2010b) reported flares from the Crab Nebula above $100 \mathrm{MeV}$ in 2009 February and 2010 September. AGILE simultaneously detected the 2010 flare (Tavani et al. 2010). Reports by the High Energy GammaRay Astronomy experiment (Aharonian et al. 2004), the Major Atmospheric Gamma-ray Imaging Cherenkov telescope (Albert et al. 2008), the High Energy Spectroscopic System (Aharonian et al. 2006), and the Very Energetic Radiation Imaging Telescope System (Wakely 2010) provide no evidence for time variability, consistent with expectations for higher energies.

Although it is extremely difficult to obtain absolute flux measurements with accuracy $\sim 1 \%$ with a single instrument, we have analyzed independent data sets from four separate operating missions. In Section 2, we present the Crab light curves measured independently by the Fermi Gamma-ray Burst Monitor (Fermi/GBM), the Swift Burst Alert Telescope (Swift/BAT), the Imager on-Board the INTEGRAL Satellite (INTEGRAL/ IBIS) and the Joint European X-ray Monitor (JEM-X), and the Rossi X-ray Timing Explorer Proportional Counter Array ( $R X T E / \mathrm{PCA})$. In Section 3, we summarize the results and discuss their implications.

\section{OBSERVATIONS AND RESULTS}

\subsection{Fermi GBM}

The GBM instrument (Meegan et al. 2009), sensitive from $8 \mathrm{keV}$ to $40 \mathrm{MeV}$, provides nearly continuous full-sky coverage via the Earth occultation technique, successfully demonstrated with BATSE (Harmon et al. 2002; Ling et al. 2000). The Harmon et al. (2002) approach has been adapted for GBM (WilsonHodge et al. 2009; Case et al. 2010). To date, six persistent and two transient sources have been detected above $100 \mathrm{keV}$ (Case et al. 2010), including the Crab.

The GBM implementation of the Earth occultation technique uses both CTIME ( 8 energy channels with 0.256 s resolution) and CSPEC data (128 energy channels with 4.096 s resolution). A detailed detector response model has been developed based

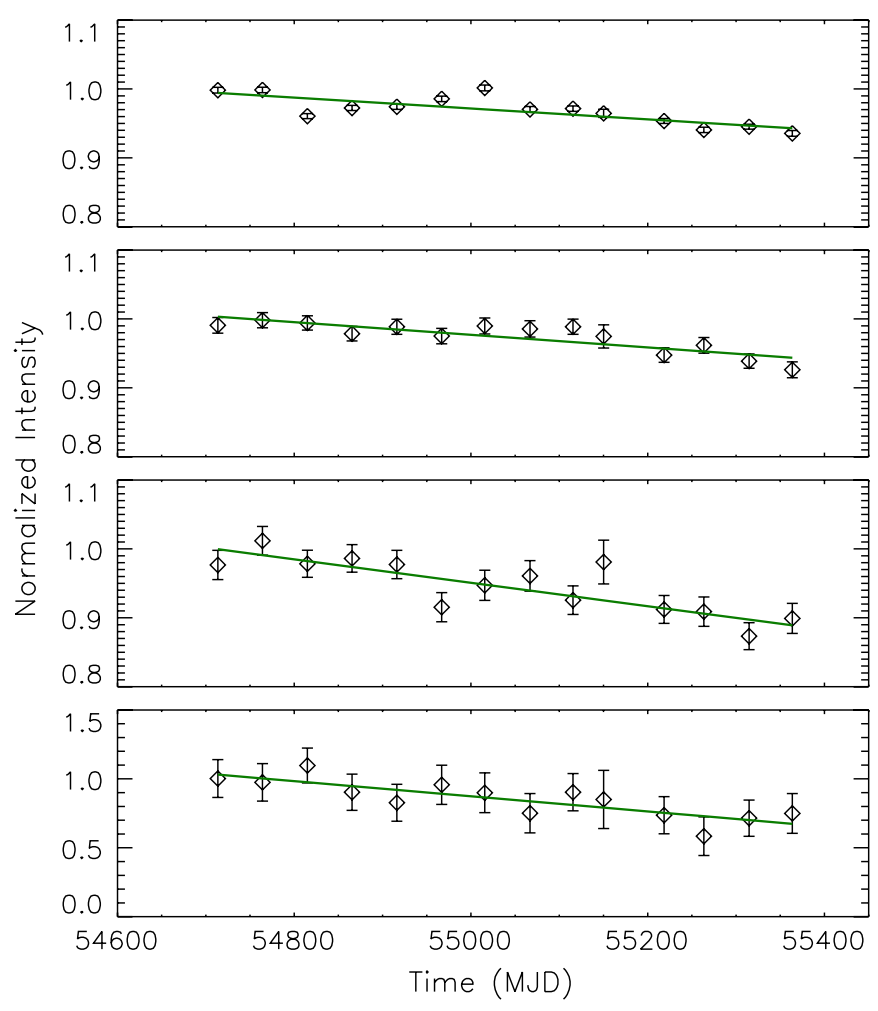

Figure 1. From top to bottom: 50 day average GBM Crab measurements for 12-50, 50-100, 100-300, and 300-500 keV. Solid lines are fits used to compute the percent change in rate.

on Geant 4 simulations confirmed by extensive ground testing in order to determine the response as a function of orientation (Hoover et al. 2008; Bissaldi et al. 2009). In flight, fits to background lines (e.g., $511 \mathrm{keV}$ ) over time show a stable gain and energy resolution in all the GBM detectors and electronics, with lines typically within $1 \%$ of their expected position.

The Crab light curve measured in four energy bands with GBM from 2008 August 12 through 2010 July 13 (MJD 54690-55390) is shown in Figure 1. With respect to the rate on MJD 54690, the Crab rate appears to have decreased steadily by more than $5 \%$ : the decrease is $5.4 \% \pm 0.4 \%, 6.6 \% \pm 1.0 \%$, $12 \% \pm 2 \%$, and $39 \% \pm 13 \%$ in the $12-50,50-100,100-300$, $300-500 \mathrm{keV}$ bands, respectively. Inclusion of a linear decline in the $12-50 \mathrm{keV}$ band improves reduced $\chi^{2}$ to $605.8 / 130=$ 4.66 from $956.3 / 131=7.30$ for a constant Crab.

\subsection{RXTE PCA}

Frequent observations with the $R X T E$ PCA were made to monitor the radio-X-ray phase of the Crab pulsed emission (Rots et al. 2004) and for calibration purposes (Jahoda et al. 2006; Shaposhnikov 2010). In the PCA, the Crab is bright $\left(\sim 2500\right.$ counts $\mathrm{s}^{-1}$ detector $\left.^{-1}\right)$. Unrejected background from all sources amounts to about $1 \mathrm{mCrab}$. The PCA is a relatively simple instrument, with commanded changes in operating conditions limited to the high voltage. Data since the last high voltage change in 1999 for PCU 2, 3, and 4 are used in this Letter.

The PCA response has two small time-dependent effects, both accounted for in the response matrices. First, xenon is slowly accumulating in the front veto layer (nominally filled with propane) and reducing the low energy sensitivity with time. Second, there is a small energy drift in the pulse height 
channel boundaries, so that a constant channel selection samples a slowly varying energy band. Both effects can influence the rate, though flux determinations (i.e., conversion of count rate to flux) account for this. In particular, the correction for changing opacity of the front veto layer is negligible in the $15-50 \mathrm{keV}$ band. Our observed changes in the Crab rate (see Figure 2) are more than five times larger than these effects combined.

Figure 2 shows total Crab rates for individual $R X T E$ pointed observations. From MJD 54690-55435 the Crab rate in PCU 2 declines by $5.1 \% \pm 0.2 \%$ and $6.8 \% \pm 0.3 \%$ in the $2-15$ and 15-50 keV bands, respectively, relative to MJD 54690. Similar results, variations of $2 \%-7 \%$, are seen if the bands are further subdivided. In spectral fits to individual PCA observations, the power-law index softens and the normalization and absorption column gradually increase with time, with no clear correlation with flux. These light curves were produced using RXTE/ PCA standard 2 data (129 energy channel, 16 s) that were extracted, background subtracted, dead-time corrected using standard RXTE recipes, ${ }^{18}$ and corrected for the known time dependence of the response.

From visual inspection of the $R X T E$ light curve, three evident peaks suggest a periodic or quasi-periodic variation with a period of 1000-1500 days. To quantify these impressions we constructed a power spectrum, shown in the third panel of Figure 2, and conducted a search for periodic signals. We averaged the corrected $15-50 \mathrm{keV}$ PCU 2 rates within uniformly spaced bins, using three bins per year, with the yearly interval where Crab cannot be observed because of Sun constraints occurring in the center of every third bin. A linear trend, which passed through the first and last binned rate, was subtracted from the rates, to limit the bleeding of low frequency power into higher frequency bands. The power spectrum was then created from the Fourier transform of the binned rates. The lower five points in the plot are from individual Fourier amplitudes, with the remainder rebinned to reduce errors. A maximum likelihood fit to the unbinned power spectrum was made using a powerlaw model. The best-fit model is shown, which has a power-law index of $2.1 \pm 0.4$.

Standard pulse search methods such as the Lomb test are inappropriate because of the underlying red noise power spectrum. The test statistic we adopted is the improvement in $\chi^{2}$ between fitting the binned rates to a quadratic and to a quadratic plus a sinusoid. The quadratic accounts for the low frequency trend in the rates. Since the source power dominates the counting statistics, we use uniform errors in the fits, setting $\sigma^{2}=P / \Delta t$, where $P$ is the power spectrum model at the middle of the region where a periodicity may be present $\left(8.5 \times 10^{-4} \mathrm{day}^{-1}\right)$ and $\Delta t$ is the bin width. As seen in the bottom panel of Figure 2, a peak in the $\Delta \chi^{2}$ is seen at $(8.5 \pm 0.7) \times 10^{-4}$ day $^{-1}$, corresponding to a period of $1180 \pm 100$ days. However, its significance is only $2 \sigma$. A longer history of the Crab flux will be needed to determine if this feature is a property of the source, or only a statistical fluctuation. Interestingly, this peak value is consistent with twice the period of $568 \pm 10$ days found in Crab radio timing noise from 1982 to 1989 (Scott et al. 2003).

The Crab pulsed flux measured using PCU 2 event mode data ( $250 \mu \mathrm{s}, 129$ energy channels, top layer) is shown in Figure 3. Although the pulsed flux (upper panel) steadily decreases at $\sim 0.2 \% \mathrm{yr}^{-1}$, consistent with the pulsar spin-down, the larger (several percent per year) variation in the signal is not seen in the pulsed emission and clearly seems to be nebular in origin.
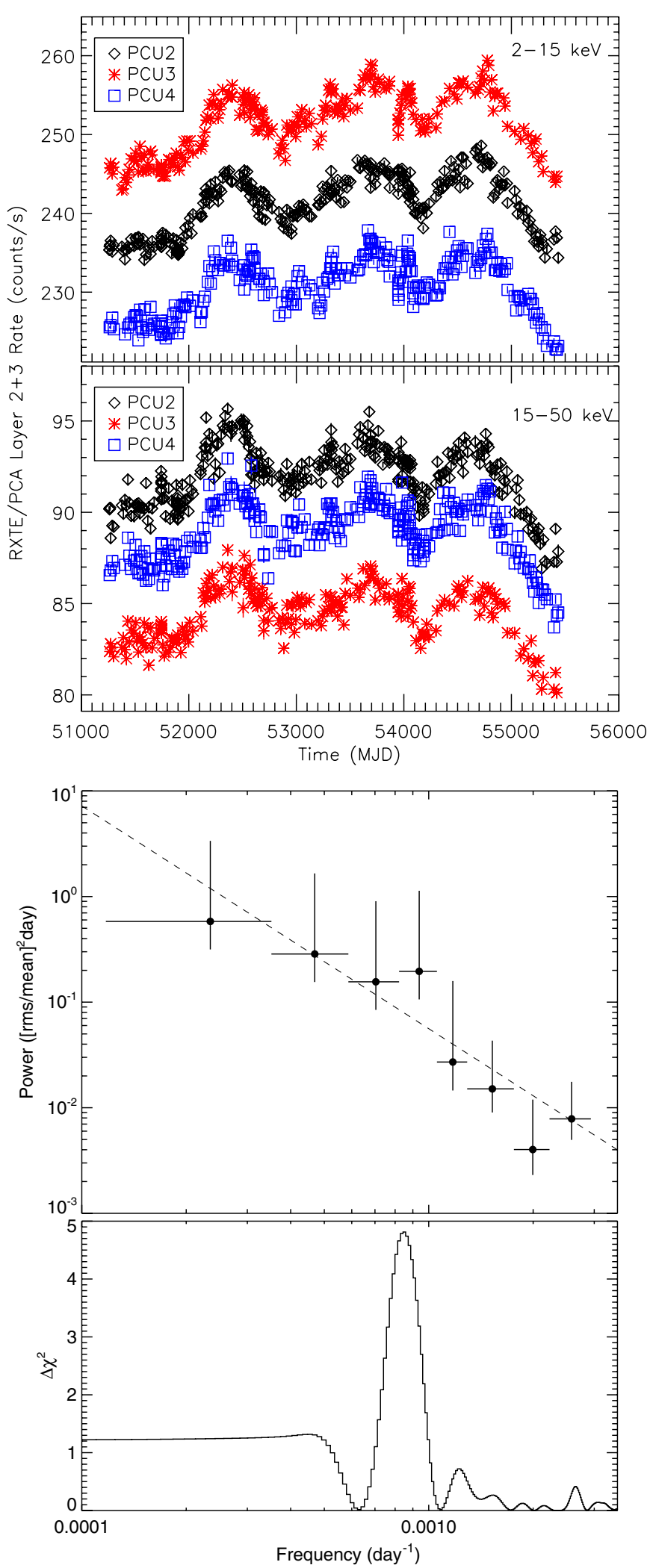

Figure 2. The upper two panels show total Crab rates (nebula + pulsar) for layers $2+3$ of PCU 2 (diamonds), PCU 3 (asterisks), and PCU 4 (squares) in the $2-15$ and $15-50 \mathrm{keV}$ bands, respectively. The third panel shows the power spectrum of the RXTE $15-50 \mathrm{keV}$ rates. The error bars give $68 \%$ confidence intervals. The dashed line is the best-fit power law. The bottom panel shows the test statistic for a search for periodic signals.

\footnotetext{
18 http://heasarc.gsfc.nasa.gov/docs/xte/recipes/
} 


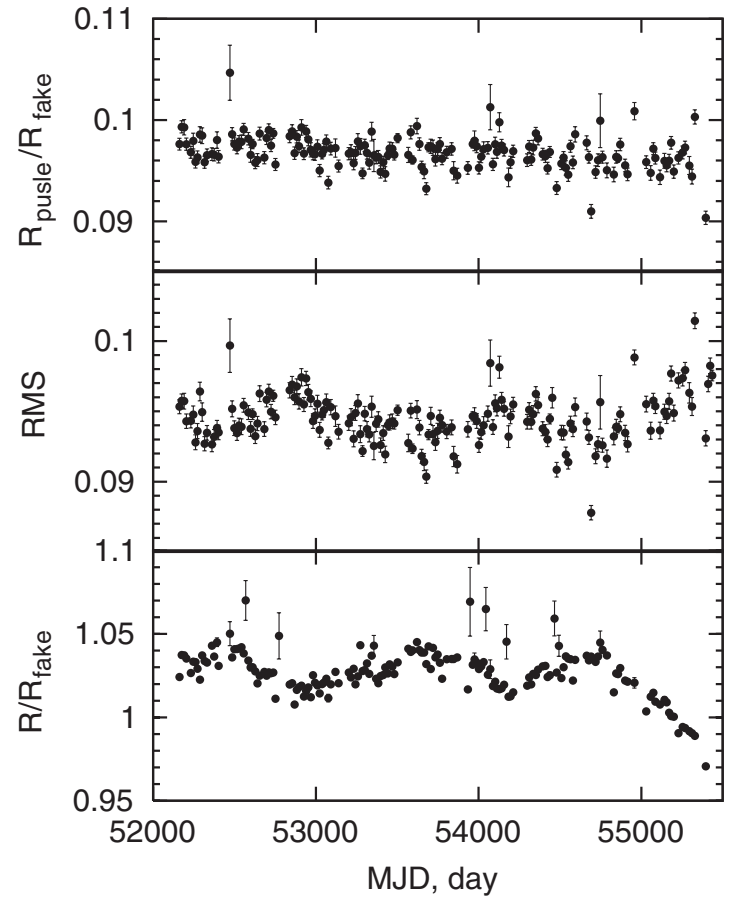

Figure 3. Top: RXTE/PCA pulsed flux (3.2-35 keV). Center: fractional rms amplitude for the first two harmonics of the pulse period. Bottom: total Crab rate in PCU 2. Rates in the top and bottom panels are normalized by the response-predicted count rate $R_{\text {fake }}$ in the $3.2-35 \mathrm{keV}$ band.

\subsection{INTEGRAL IBIS and JEM-X}

Here, we present results from the JEM-X (3-35 keV; Lund et al. 2003) and the INTEGRAL Soft Gamma-Ray Imager layer of IBIS (ISGRI, $15 \mathrm{keV}-10 \mathrm{MeV}$; Ubertini et al. 2003) on board INTEGRAL (Winkler et al. 2003). The Crab has been observed every spring and fall with INTEGRAL since 2002, mainly for calibration purposes. To reduce systematic effects as much as possible, we have selected on-axis $(<0.25)$ observations for JEM-X2 and observations within $10^{\circ}$ of on-axis for IBIS/ ISGRI. We include only JEM-X data using the latest on board software (since MJD 53068).

In Figure 4, ISGRI and JEM-X2 count rates from individual pointings averaged over the 3 day INTEGRAL orbit with rms errors are shown. The ISGRI data were analyzed with the Off-line Analysis (OSA) package (Courvoisier et al. 2003) version 9 with the settings used for the INTEGRAL Galactic Bulge monitoring program ${ }^{19}$ light curves (Kuulkers et al. 2007). Using images from individual pointings, the point-spread function of ISGRI is fitted. These images are integrated in a given energy band after gain, offset, and charge loss corrections are performed for each event. A time-dependent effective area correction, usually performed by assuming that the Crab flux is constant, has been excluded from these data, meaning that not all systematic effects are taken into account. Known effects include residuals in gain and charge loss corrections, present with an amplitude of $\sim 1 \%-2 \%$, varying on month-years timescales. Similarly, for JEM-X, the ad hoc piecewise linear correction (added to OSA to reduce time trends in the Crab flux) was excluded from the standard OSA analysis. JEM-X consists of two identical units: JEM-X1 and JEM-X2. During the period of interest, JEM-X2 has mostly been in standby mode and JEM-X1 the active unit. A gradual decrease has been observed in the sensitivity of

\footnotetext{
19 http://integral.esac.esa.int/BULGE/
}

JEM-X1, so only JEM-X2 is shown. The scatter in the JEM-X data is large compared to the observed Crab variations, especially below $10 \mathrm{keV}$. From MJD 54690-55390, the ISGRI $15-50,50-100$, and $100-300 \mathrm{keV}$ flux decreases by $8.2 \% \pm 1.1 \%, 8.3 \% \pm 1.1 \%$, and $5.7 \% \pm 1.0 \%$, respectively, relative to MJD 54690.

\subsection{Swift BAT}

Swift/BAT is a coded aperture telescope operating in the 14-150 keV range (Barthelmy et al. 2005). The Swift/BAT 14-50 and 50-100 keV light curves (see Figure 5) are based on publicly available 58 month light curves $^{20}$ from the Swift/BAT all-sky hard X-ray survey (Tueller et al. 2010; Baumgartner et al. 2010) extended to 2010 May 30 by the BAT team. We binned data from individual Swift pointings in 50 day intervals, eliminating pointings of less than $200 \mathrm{~s}$ duration and those in which less than $15 \%$ of the BAT detectors were illuminated by the Crab. The statistical errors on each data point are small $(0.1 \%)$ and are dominated by systematic errors. We estimate the systematic errors to be $\sim 0.75 \%$ by assuming that the longterm variations in the light curve are due to real variations in the Crab, and that the shorter term variations around that trend are representative of the systematic error. The BAT data show variations in the Crab flux at the level of $\sim 3 \% \mathrm{yr}^{-1}$. From MJD $54690-55340$, BAT observes a decrease of $6.7 \% \pm 0.7 \%$ and $10.4 \% \pm 0.8 \%$ in the $15-50$ and $50-100 \mathrm{keV}$ bands, respectively, relative to the rate on MJD 54690, similar to the decrease seen by GBM in the same energy range.

\section{DISCUSSION AND SUMMARY}

Figure 5 shows composite light curves combining the overlapping results from RXTE, INTEGRAL, Swift, and Fermi/GBM. All instruments agree well from 2008 to 2010, with all instruments registering a decline in the Crab $15-50 \mathrm{keV}$ flux of $\sim 7 \%$ (70 mCrab) over the 2 years starting at MJD 54690, with a similar decline in the 50-100 keV band. PCA and BAT continue to agree back to the start of the Swift mission. For RXTE, Swift, and INTEGRAL/ISGRI the latest measurements shown are significantly below previous minima. INTEGRAL/ISGRI shows evidence for the dip near MJD 54100-54200 and the increase before $\sim$ MJD 53700, with similar but less significant variations seen in JEM-X2. Prior to this time, the PCA measurements show continued variations extending back to $\sim \mathrm{MJD}$ 52000 , which are not seen with ISGRI in the $20-50 \mathrm{keV}$ band. We investigated the effect of a change in the default dithering pattern since 2006 March, but found that this cannot explain the observed difference. Known systematic errors in ISGRI energy reconstruction are expected to account for $\sim 1 \%-2 \%$ deviations. Beginning at $\sim$ MJD 54000 , there is a strong correlation among the results from the four independent instruments with very different signal-to-noise characteristics and observing techniques: Earth occultation, coded-mask imaging, and collimated detectors. The range of techniques strengthens the case that the variation is intrinsic to the Crab. We found no apparent correlations between these variations and variations in the INTEGRAL/SPI anti-coincidence detector count rates or GBM count rates, disfavoring local background condition changes as a possible origin, and further supporting a Crab origin. The pulsed flux stability suggests that the observed variations are nebular.

The observed time variability may be explained by models of the Crab pulsar wind flow. In some models (e.g., Camus et al.

\footnotetext{
20 http://swift.gsfc.nasa.gov/docs/swift/results/
} 


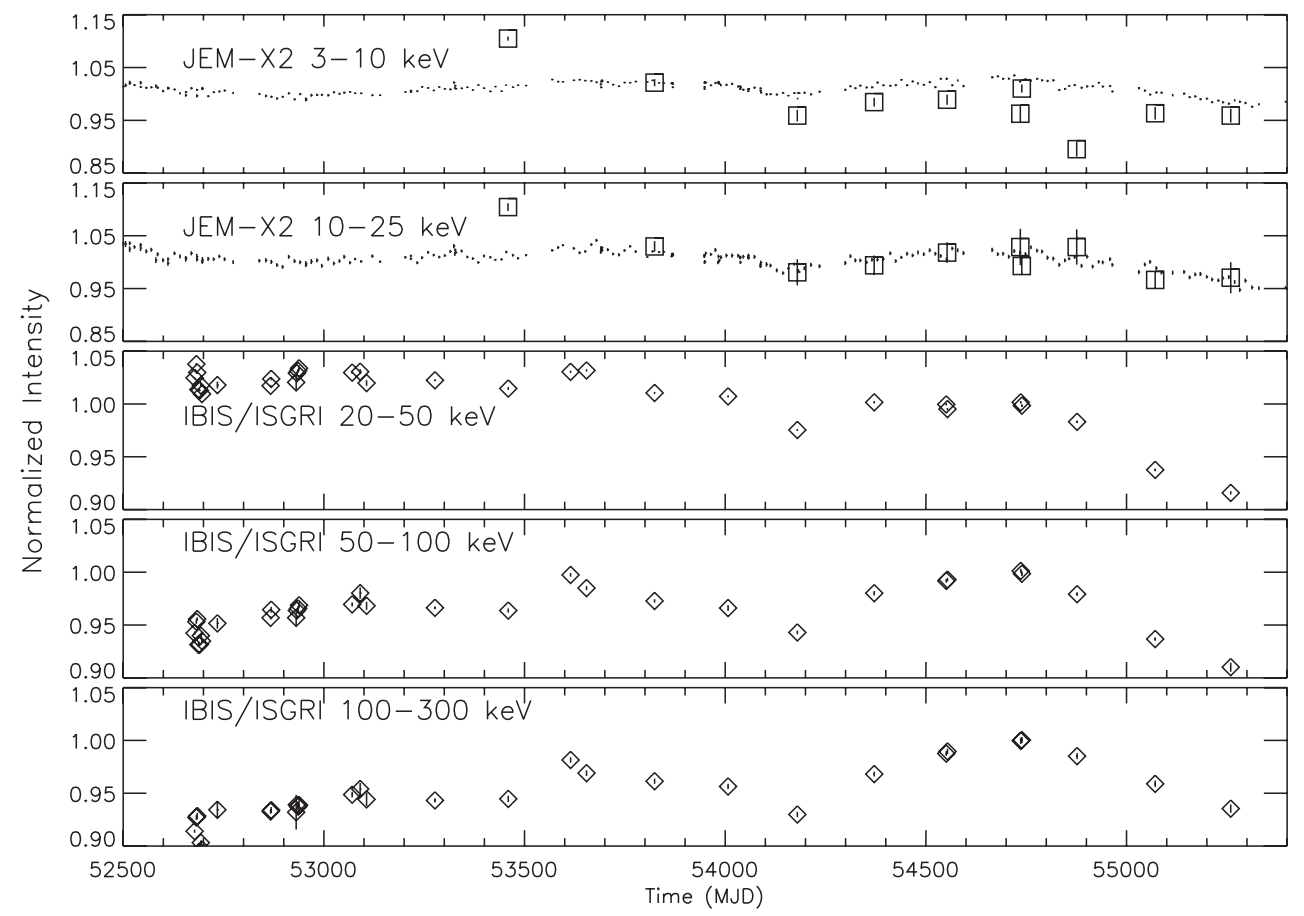

Figure 4. INTEGRAL 3 day averaged light curves of the Crab measured in the 3-10 and 10-25 keV bands with JEM-X, and the 20-50, 50-100, and 100-300 keV bands with ISGRI. Normalized RXTE PCU2 rates in the 2-15 and 15-50 keV bands are overplotted with the JEM-X data for comparison.

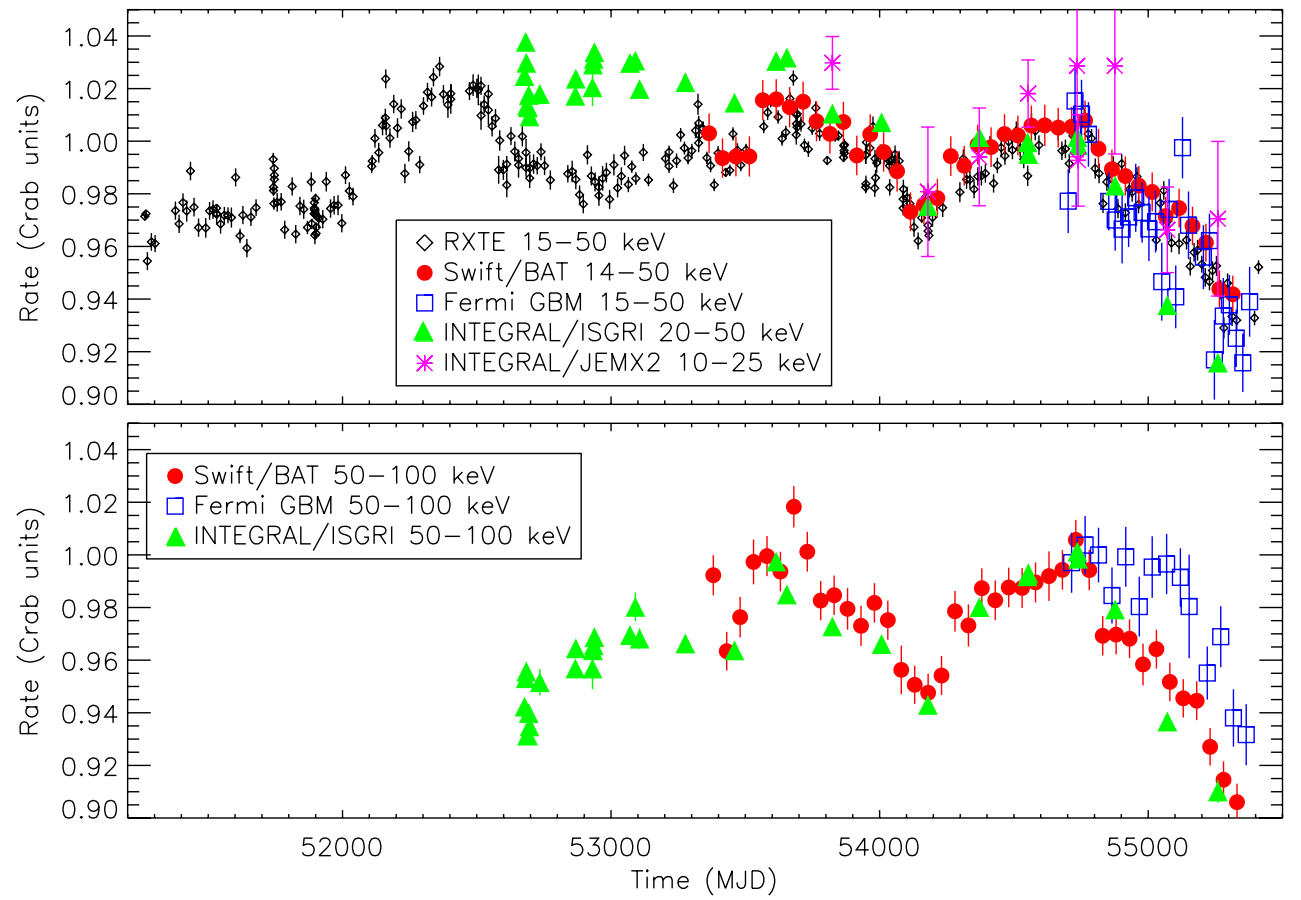

Figure 5. Composite Crab light curves for RXTE/PCA (15-50 keV: black diamonds), Swift/BAT (top: 14-50 keV, bottom: 50-100 keV: red filled circles), Fermi/GBM (top: $15-50 \mathrm{keV}$, bottom: 50-100 keV: open blue squares), INTEGRAL/ISGRI (top: 20-50 keV, Bottom: 50-100 keV: green triangles), and INTEGRAL/JEM-X2 $(10-25 \mathrm{keV})$. Each data set has been normalized to its mean rate in the time interval MJD 54690-54790. All error bars include only statistical errors.

2009), a radial plasma flow in the equatorial plane decelerates downstream of a termination shock located at a radius of about $0.5 \mathrm{lt}-\mathrm{yr}$ and near the inner ring observed in X-rays (Weisskopf et al. 2000). Due to adiabatic and synchrotron losses in the fluid the flow becomes inhomogeneous with large variations in local magnetic field strength. These magnetosonic waves are relativistic and the variability timescale is roughly the fluid crossing time across the shock diameter or 1-2 years. Alternatively (Spitkovsky \& Arons 2004), variability on scales of the ion Larmor radius may result from cycles of compression of the electron-positron plasma induced by magnetosonic waves caused by the cyclotron instability in the ion orbits.

Chandra (Weisskopf et al. 2000; Mori et al. 2004) and XMMNewton (Kirsch et al. 2006) observations of the Crab suffer from pileup effects, making it difficult to monitor absolute fluxes at the level of a few percent. No Chandra ACIS observations of the Crab were performed from MJD 54135-55466. Nevertheless, both instruments have shown that the spectrum of the 
synchrotron X-rays grows distinctly softer as distance from the pulsar increases. Since higher energy electrons have shorter synchrotron lifetimes, the spectrum becomes softer as the particles move outward and synchrotron losses grow. Alternatively, the site of the main particle acceleration or the spectral steepening as a function of distance from the pulsar and shock region may vary with time.

The differential photon spectrum $d N / d E$ produced by synchrotron-emitting electrons depends on magnetic field strength $B$ and photon energy $E$ as $d N / d E \sim B^{\gamma} E^{-\gamma}$, where $\gamma$ is the power-law photon energy index (Felten \& Morrison 1966), suggesting that the observed change in flux could be produced either by a change in the accelerated electron population or a change in the nebular magnetic field of a few percent.

In summary, the widely held assumption that the Crab can be used as a standard candle, suitable for normalizing instrument response functions and for calibrating X-ray instruments, should be treated with caution. Although obtaining absolute calibrations and instrument normalizations at $\sim 1 \%$ is difficult, the results presented here from four independent spacecraft demonstrate that in fact the nebular X-ray/gamma-ray emission from the Crab varies at a level of $\sim 3.5 \% \mathrm{yr}^{-1}$. The variation is seen in the nebular emission, and so apparently results from changes in the shock acceleration or the nebular magnetic field. We cannot predict if the present decline will continue or if the $\sim 3$ year pattern will persist. Longer baselines and multi-wavelength observations are needed to answer these questions.

This work is supported by the NASA Fermi Guest Investigator program, NASA/Louisiana Board of Regents Cooperative Agreement NNX07AT62A (LSU), the Louisiana Board of Regents Graduate Fellowship Program (J.C.R.), and the Spanish Ministerio de Ciencia e Innovación through the 2008 postdoctoral program MICINN/Fulbright under grant 20080116 (A.C.-A.). This research has made use of data obtained through the High Energy Astrophysics Science Archive Research Center Online Service, provided by the NASA/Goddard Space Flight Center; public $S$ wift/BAT results made available by the Swift/BAT team; and observations with INTEGRAL, an ESA project funded by ESA member states (especially the PI countries: Denmark, France, Germany, Italy, Switzerland, Spain) and Poland, and with the participation of Russia and the USA.

\section{REFERENCES}

Abdo, A. A., et al. 2010a, ApJ, 708, 1254

Abdo, A. A., et al. 2010b, Science, submitted (arXiv:1011.3855)

Aharonian, F., et al. 2004, ApJ, 614, 897
Aharonian, F., et al. 2006, A\&A, 457, 899

Albert, J., et al. 2008, ApJ, 674, 1037

Aller, H. D., \& Reynolds, S. P. 1985, ApJ, 293, L73

Barthelmy, S. D., et al. 2005, Space Sci. Rev., 120, 143

Baumgartner, W., et al. 2010, ApJS, submitted

Bietenholz, M. F., Frail, D. A., \& Hester, J. J. 2001, ApJ, 560, 254

Bissaldi, E., et al. 2009, Exp. Astron., 24, 47

Camus, N. F., Komissarov, S. S., Bucciantini, N., \& Hughes, P. A. 2009, MNRAS, 400, 1241

Case, G. L., et al. 2010, ApJ, submitted (arXiv:1009.4953)

Courvoisier, T.J.-L., et al. 2003, A\&A, 411, L53

De Jager, O. C., Harding, A. K., Michelson, P. F., Nel, H. I., Nolan, P. L., Sreekumar, P., \& Thompson, D. J. 1996, ApJ, 457, 253

Felten, J. E., \& Morrison, P. 1966, ApJ, 146, 686

Greiveldinger, C., \& Aschenbach, B. 1999, ApJ, 510, 305

Harmon, B. A., et al. 2002, ApJS, 138, 149

Hester, J. J. 2008, ARA\&A, 46, 127

Hester, J. J., et al. 1995, ApJ, 448, 240

Hester, J. J., et al. 2002, ApJ, 577, L49

Hoover, A. S., et al. 2008, in AIP Conf. Proc. 1000, Gamma-Ray Bursts 2007, ed. M. Galassi, D. Palmer, \& E. Fenimore (Melville, NY: AIP), 565

Jahoda, K., Markwardt, C. B., Radeva, Y., Rots, A. H., Stark, M. J., Swank, J. H., Strohmayer, T. E., \& Zhang, W. 2006, ApJS, 163, 401

Jourdain, E., \& Roques, J. P. 2009, ApJ, 704, 17

Kirsch, M. G. F., et al. 2005, Proc. SPIE, 5898, 22

Kirsch, M. G. F., et al. 2006, A\&A, 453, 173

Kuulkers, E., et al. 2007, A\&A, 466, 595

Ling, J. C., \& Wheaton, W. A. 2003, ApJ, 598, 334

Ling, J. C., et al. 2000, ApJS, 127, 79

Lund, N., et al. 2003, A\&A, 411, L231

Meegan, C., et al. 2009, ApJ, 702, 791

Meyer, M., Horns, D., \& Zechlin, H.-S. 2010, A\&A, 523, A2

Mori, K., Burrows, D. N., Hester, J. J., Pavlov, G. G., Shibata, S., \& Tsunemi, H. 2004, ApJ, 609, 186

Much, R., et al. 1995, A\&A, 299, 435

Ng, C.-Y., \& Romani, R. W. 2006, ApJ, 644, 445

Reynolds, S. P., \& Chevalier, R. A. 1984, ApJ, 278, 630

Rots, A., Jahoda, K., \& Lyne, A. G. 2004, ApJ, 605, 129

Scott, D. M., Finger, M. H., \& Wilson, C. A. 2003, MNRAS, 344, 412

Shaposhnikov, N. 2010, in Presentation at the 2010 Meeting of the IACHEC, Woods Hole, http://web.mit.edu/iachec/meetings/2010/index.html

Smith, M. 2003, MNRAS, 346, 885

Spitkovsky, A., \& Arons, J. 2004, ApJ, 603, 669

Tavani, M., et al. 2010, ATel, 2855

Toor, A., \& Seward, F. D. 1974, AJ, 79, 995

Tueller, J., et al. 2010, ApJS, 186, 378

Ubertini, P., et al. 2003, A\&A, 411, L131

Verrecchia, F., in't Zand, J. J. M., Giommi, P., Santalomazza, P., Granata, S., Schuurmans, J. J., \& Antonelli, A. 2007, A\&A, 472, 705

Wakely, S. P. 2010, in Proc. VERITAS Workshop on High Energy Galactic Physics, ed. E. Aliu, J. Holder, \& R. Mukherjee (New York: Columbia Univ.), eConf C1005281, 2

Weisskopf, M. C., Guainazzi, M., Jahoda, K., Shaposhnikov, N., O’Dell, S. L., Zavlin, V. E., Wilson-Hodge, C., \& Elsner, R. F. 2010, ApJ, 713, 912

Weisskopf, M. C., et al. 2000, ApJ, 536, L81

Wilson-Hodge, C. A., et al. 2009, in Proc. Fermi Symp., eConf C091122, arXiv:0912.3831

Winkler, C., et al. 2003, A\&A, 411, L1 